\title{
Impacts of Multiple Color Nominal Coding on Usefulness of Graph Reading Tasks
}

\author{
Gia Kim \\ School of Business (Information Technology) \\ James Cook University \\ Cairns, Australia \\ gia.kim@jcu.edu.au
}

\author{
Siu Man Lui \\ School of Business (Information Technology) \\ James Cook University \\ Cairns, Australia \\ carrie.1ui@jcu.edu.au
}

\begin{abstract}
Multiple line graphs have been extensively used in business, finance and education. Previous color researches suggested that high contrast may be useful for nominal information coding in information design. However, empirical studies on its impacts on the graph-reader's perception towards usefulness of the multiple line graph have been rarely examined. This study conducted an experiment to evaluate graphs using two different color combinations. The participants perceived the high luminance contrast Red-Green-Blue graph is more useful than the low luminance contrast Orange-Green-Cyan graph for complicated tasks using multiple lines. There was no difference of perceived usefulness across simple tasks including using a single line. Despite of the results, participants' actual task completion time shows there is no difference in terms of graph reading complexity between the two different color contrast groups.
\end{abstract}

\section{INTRODUCTION}

Graphical presentation has been used for decades in business and everyday life to convey information effectively. Annual reports of $83 \%$ of Australian listed companies illustrate graphs conveying financial or non-financial variables and similarly, $73 \%$ of not-for-profit organizations' annual reports include graphs [1]. Penrose [2] reported that more than $80 \%$ of annual reports across countries use graphs, including $92 \%$ for the U.S reports.

Visual charts are also extensively used in the financial industry. Users of annual reports, including trained financial analysts and non-professional individual investors, may spend only fifteen minutes on looking at the annual report during their decision making process [2]. Indeed, they often look at only financial charts to make their decision. This shows financial graphics play an important role in interpreting their desired information and the usage of graphs remains massive in daily life. Despite increased research attempting to understand the nature of graph interpretation [3], the contributory factors that design effective graphs still remain as questions.

Color is a fundamental element in the design of diagrams, charts, and information visualization [4]. Misguided colors may cause the reader to interpret information incorrectly, whereas well-targeted colors can enhance the reader's processing of graphic information, thereby increasing the reader's processing performance [5]. For example, subsequent researchers variegated the traditional 'Stroop Effect' by testing the reader's interpretive, time-perception skills in reading the misguided word 'blue' as an orange color against the same well-targeted word as a blue color $[6,7]$. The blue-orange association required a longer recognition time.

Over time, a color reader acquires a 'color vocabulary'. Here, certain colors have near universal acceptance. Green indicates uptrend and red indicates downtrend in the financial market, and in general, green means 'Go' and red means 'Stop'. Hence, the role of color indication is of importance in visual communication design. In fact, many prior researchers revealed their concerns about misguided graph design through graph types, colors, scale, emphasis, and size that may mislead graph-users and can cause them to make different decisions [2].

To date, consideration of color theories has had low significance in the design of information system solutions, and system developers have generally resorted to the Red-GreenBlue combination to match with three data values [4]. This leads readers or computer end-users to become 'habituated' and to accept without-question whatever colors are set as default.

In financial displays, a white or light-tone background with blue or red lines remains a traditional representation for line graphs. The Australia Stock Exchange price charts use RGB combinations [8]. Slight expressive color combinations to this fixed color pattern have emerged with Google Finance [9] using red-blue-amber lines for and Bloomberg [10] using a blue background with a white line. Thus, existing information systems reveal inconsistencies in rules to select effective color labels for charts.

Furthermore, the usability of color combinations for graphs has rarely been examined in the graph reading context. For the past decades, general color-pair studies [11] and comparisons between mono- and multiple color graphs [12] have been conducted but empirical graph studies on multiple color combination have seldom been conducted. Considering excessive uses of multiple line graphs in business, education, and the financial industry, empirical color examinations in graph comprehension is crucial.

Hence, this study aims to examine the effect of the color selection for the multiple lines of a graph on the graphreader's perception towards the usefulness of the graph when interpreting information from the graph. 
Another focus of this study is to investigate the effect of task types as a moderator on the impacts of color combination. Researchers have focused on the effect of the different levels of task types on graphical presentation [13, 14], but this impact has rarely been examined in color graphs. Here, the research question of this study is how color combination influences the graph reader's perceived usefulness towards graph comprehension depending on task types.

In the next section, color theories pertinent to this study are discussed. The hypotheses based on the literature review are then developed, and the research design and the findings from the experiment are introduced. In the final section, this study concludes by guiding future research agenda and recommendations to practitioners are suggested.

\section{THEORETICAL BACKGROUND}

Fundamental uses of color in information displays are believed to label items, to measure quantity in informative maps, to represent or imitate reality in such as visualization, and lastly for beauty that is to add attractive values to the display [4, 15]. Among these purposes of color-enhanced information presentation, a vital role of color in graphs is most importantly for labeling.

The technical name for labeling in color science is called nominal information coding [5]. A nominal code of graphs is required to be remembered and recognized. Color may be highly effective as a nominal code. Color researchers have suggested designers to pick high contrast colors for nominal coding in information displays [16]. Tufte [15] argued that color labels distinguish an object from another in information design by matching with distinctive colors. Ware [5] also suggested seven guidelines, including distinctness and contrast, in selecting a set of color labels.

Previous color studies seem to simply assume high color contrast is the best way of information labeling, but have rarely considered varied specific task types of graph reading context. This section will discuss the ways of creating maximum discrimination for nominal coding in literature review. In addition, other considerations when choosing a color set for this multiple-line graph experiment will be discussed based on literature review with physiological and psychological color aspects.

\section{A. Physiological Color}

Color contrast is defined as a color appearance phenomenon that causes stimuli to shift in color appearance to the opponent dimensions when interacting with the color of their neighbor or background [17]. In other words, a light background induces a stimulus to appear darker, a red color induces green appearance, a yellow induces blue, and so on. In order to understand color contrast phenomenon, color researchers have discovered the principles of human vision sensation.

According to trichromacy theory, human color vision senses three wavelengths of red, green, and blue, with peaks at wavelengths of approximately 580, 540, and 440 nanometers respectively [18]. The hue of a color is sensed in a unique combination these three receptor classes of the retina produce.
The lens of the human eye becomes more convex to focus on a red color, as if an object nearer to the viewer $[5,18]$. To focus on a blue color, the lens becomes more relaxed, as if for an object farther away from the viewer. Thus, the mixture of advanced colors (red-yellow family) and receded colors (blueindigo family) for a labeling set of information displays easily creates hue color contrast.

Since Hering [19] proposed the theory that there are six elementary colors of red-green, yellow-blue, and black-white and these three axes are perceived as opponent pairs, the modern opponent process theory has been established on physiological basis of two distinct wavelength and a luminance channels. These six colors are believed to have unique hues, and they are often used in basic nominal coding, followed by orange, cyan, pink and gray as the top ten unique hues recommended for use in labels [5].

Another consideration of using unique hues is the relationship between the viewer's perceived visual stress and color contrast. The viewer may perceive high color contrast information displays useful to conduct tasks but this high contrast also may cause visual stress. Color scientists reveal that areas of stron-tone colors, such as strong blue and strong red, and high contrast can generate afterimages. As a result, the viewer may experience visual stress from prolonged viewing when looking away from the screen [18].

In addition to chromatic contrast, Ware [5] recommenced significant luminance contrast to display codes. Luminance is a physical measure to define an amount of light in the visible region [5]. Luminance is sometimes believed another factor causing visual stress. Objects with radiating zones or auras have attracted human visions, human visual perception has been found to be more sensitive to bright light than dull light or low illumination objects [20].

However, the high luminance values may be too bright and stressful on the eye when applied to digital monitors. Hence, when viewing computer screen colors, the viewer may experience painful eye sensations when looking at bright colors such as cyan and green, and so may withdraw from this information displays. The level of visual stress is likely to affect the graph-reader's performance.

\section{B. Psychological Color}

Gestalt psychologists specified fundamental perceptual phenomena of the way the viewer observes patterns in visual displays [5]. One of the phenomena is that similar elements tend to be grouped together $[5,18]$. Analogous hues, sequential tones, and little luminance difference are most likely to be perceived as a group when they reside in the neighborhood against a dissimilar color in the display.

On the other hand, a unique color different against its surrounding generates a 'pop-out' effect [21]. Prior visual search studies show users perform effectively faster when whey obtain information of items with a salient feature [22]. A salient feature is, in fact, one of the essential factors for nominal codes in making information contents easier to interpret. A vivid color among pale items, or a dull color among multiple vivid tones is an example of salient features. 
When opponent colors, such as red-green and blue-yellow, are used all together on the same screen, less salient features are expressed because this contrast may disturb the viewer's perception of the items as a group. Regardless the level of luminance, when the difference of two items' luminance is little, relatively the third item displaying luminance difference is more likely to pop out.

Two types of discernibilities are required for nominal information coding. First, how easy it is to distinguish an item from its background. Second, how easy it is to distinguish an item from its neighbor, that is, another item. The luminance (98) of a yellow object on the luminance (100) of a white background displays low legibility, and thus, designers tend to not to use a yellow color for a label on a white background. Hence, the classical RGB combination represents not only unique hue contrast, but also higher distinctiveness between items and a white background and higher distinctiveness among the RGB items, than any other color combination.

The discussion of discernibilities for labels naturally raises the issue of the ideal number for use for labeling. Estimates for the most effective number to display category information vary among color researchers. McDonald [18] suggests designers to limit the number to seven or less. Ware [5] states five codes can be rapidly perceived and maximum ten codes may be acceptable. The ideal color sets for the variation of the number may depend on tasks of the way to extract information from the display, and empirical evidences have been rarely demonstrated.

In addition to the number of color codes, the size of colorcoded area is a major factor that affects the viewer's perception of information design [5]. In general, color-coded small objects, such as color lines of a graph, should present strong and highly saturated colors for maximum discrimination. The color presentation rules for lines should be applied differently from the ones for shapes. Most of the previous color comparisons have been studies based on shapes that illustrate reasonably large field size.

In fact, according to trichromatic theory, a red square is perceived bigger and alerts more attention on a white background, while a blue square looks smaller and further than actual [18]. On the other hand, a blue thin line can be perceived clearer and more obvious on a white background, compared to a green line perceived much more spread, less clear and less obvious.

This color perception can be changed depending on its neighboring items. A cyan square looks bigger than an orange square on a white background due to its luminance contrast against white. However, an orange thin line neighboring a cyan line can be perceived clearer and more obvious than a cyan line that looks more spread against a white background.

An orange line neighboring dark-tone color items can look more spread because the dark-tone line can be seen more obvious compared to the orange line. This color perception may affect the graph-reader's performance with the graph and their perception about whether the information display useful or not, for their specific tasks.

\section{HYPOTHESES DEVELOPMENT}

This study examines the effect of the color sets that present high and low luminance contrast on the graph-reader's perception towards usefulness of the graph when conducting designed tasks of interpreting information from the graph.

\section{A. Color Luminance Contrast}

The color codes for two graphs are selected in terms of unique colors [5], color hue contrast, luminance contrast [4], difference against background, and difference between lines [18]. The selected color sets represent high luminance contrast and low luminance contrast overall. The RGB combination consists of the primary unique colors of red $(1,0,0)$, green $(0$, $1,0)$, and blue $(0,0,1)$, while the Orange-Green-Cyan combination consists of non-unique colors but still remains as meaningful selects for information displays generating from the primary colors' mixture. A green $(0,1,0)$ remains the same, a cyan $(0,1,1)$ is generated from a mixture of green $(0$, $1,0)$ and blue $(0,0,1)$, and an orange $(1,0.5,0)$ is substituted for a yellow $(1,1,0)$ coming from a mixture of red $(1,0,0)$ and green $(0,1,0)[4,18]$.

Both RGB and OGC sets present high hue contrast where the three hues are located as approximately 120 degree in the color wheel showing a triad-primary combination [23]. This hue contrast may be satisfied at the minimum requirement for information display labels to distinguish one from another.

The manipulation of luminance contrast is divided into the difference of a line against a white background and the difference between a line and its neighboring line [5, 18]. In short, the RGB color combination represents high luminance contrast while the OGC combination represents low luminance contrast. The OGC combination, compared to RGB, consists of expressive colors presenting vivid tones. All the three colors of OGC are selected high luminance colors that show at least more than 70 of the luminance value, as shown in Table I.

TABLE I

LUMINANCE MANIPULATION FOR THE EXPERIMENT

\begin{tabular}{|c|c|c|c|c|}
\hline Coding & RGB (hex.) & Luminance & OGC (hex.) & Luminance \\
\hline Background & $\begin{array}{c}\text { White } \\
(\# 000000)\end{array}$ & 100 & $\begin{array}{c}\text { White } \\
(\# 000000)\end{array}$ & 100 \\
\hline \multirow[t]{3}{*}{ Lines } & $\begin{array}{c}\text { Red } \\
(\# F F 0000)\end{array}$ & 54 & $\begin{array}{c}\text { Orange } \\
(\# \text { FF9900) }\end{array}$ & 73 \\
\hline & $\begin{array}{c}\text { Green } \\
(\# 00 \mathrm{FF} 00)\end{array}$ & 88 & $\begin{array}{c}\text { Green } \\
(\# 00 \text { FF00) }\end{array}$ & 88 \\
\hline & $\begin{array}{c}\text { Blue } \\
(\# 0000 \mathrm{FF})\end{array}$ & 30 & $\begin{array}{c}\text { Cyan } \\
(\# 00 \text { FFFF })\end{array}$ & 91 \\
\hline \multirow{4}{*}{$\begin{array}{l}\text { Difference } \\
\text { Between } \\
\text { Lines }\end{array}$} & $\mathrm{R}-\mathrm{G}$ & 34 & $\mathrm{O}-\mathrm{G}$ & 15 \\
\hline & $\mathrm{R}-\mathrm{B}$ & 24 & $\mathrm{O}-\mathrm{C}$ & 18 \\
\hline & $G-B$ & 58 & $\mathrm{G}-\mathrm{C}$ & 3 \\
\hline & Mean & 39 & Mean & 12 \\
\hline \multirow{4}{*}{$\begin{array}{c}\text { Difference } \\
\text { Background } \\
\text { and Line }\end{array}$} & $\mathrm{W}-\mathrm{R}$ & 46 & $\mathrm{~W}-\mathrm{O}$ & 27 \\
\hline & $\mathrm{W}-\mathrm{G}$ & 12 & $\mathrm{~W}-\mathrm{G}$ & 12 \\
\hline & $\mathrm{W}-\mathrm{B}$ & 70 & $\mathrm{~W}-\mathrm{C}$ & 9 \\
\hline & Mean & 43 & Mean & 16 \\
\hline Sum of L.D & \multicolumn{2}{|c|}{244} & \multicolumn{2}{|c|}{84} \\
\hline Mean & \multicolumn{2}{|c|}{41} & \multicolumn{2}{|c|}{14} \\
\hline
\end{tabular}


According to our pilot test in 2009, low luminance combination (dark-tone lines) for nominal codes for a line graph presented lower legibility than high luminance combination (vivid-tone lines). This time, this color luminance experiment is only focus on a vivid-tone combination.

In general, the extracted luminance values of fundamental colors range from a white color at 100 , yellow at 98 , cyan at 91 , green at 88 , magenta at 60 , red at 54 , blue at 30 , and black is equal to 0 (derived from Adobe Photoshop color picker). These values show yellow, cyan, and green are considered stimulating computer screen colors that evoke perceptions of brightness, and so the user is more likely to be alert to cyan and green, than low luminance of color, such as blue. Hence, orange, green, and cyan are strategically selected to present high luminance of vivid-tone but low luminance contrast between lines as well as against a white background, as illustrated in Table I.

Table I shows the difference of the luminance between lines of the RGB graph (39) is much higher than the OGC graph (12). A green color and a cyan are distinguishable due to different hues on the screen but they both radiate high luminance on the screen and the difference between two is little. Furthermore, the luminance difference between the background and a line color of the RGB is also much higher (43) than the OGC (16). Thus, on average, the RGB color set for a line graph clearly presents much higher luminance contrast (41) than the OGC graph (14). The OGC combination looks rather harmonized as a group.

\section{B. Task Type}

Task complexity is believed to increase when there are more information cues to convert or more steps to execute [24]. Information cues in graphs derive grid systems with values. Processing actions are required ranging from simple steps of observing graph trends to complicated steps of extracting numerous values.

Task types in graphs comprehension are normally divided into information retrieval systems that require specific extractions and other information retrievals that contains nonspecific general task values applicable to displays [13]. A question like "Is the trend of Line X going up or down?" is a general question that does not require any specific value.

Depending on the level of task complexity, elementary graph-reading tasks usually lead a simple-step of mental workload, such as reading off the value from the graph like "What is the value of Line X?". Comparatively advanced tasks require multiple combined processes involving more complicated mental workload. A question "For the period of $15^{\text {th }}$ to $30^{\text {th }}$ April, what is the difference between the range of Stock X and Stock Y?" requires combined searching and reading processes and then calculation using those values.

In terms of color graph comprehension, effective color selection for the graph may differ relying on the task complexity. The most complicated task for this experiment asks the graph-reader to compare extracting values from two lines. The two lines intersect over the trend and although the reader is not required to use the third line, this line's several intersections with the two target lines may distract the reader's performance. This study hypothesizes that the high luminance contrast of the RGB graph is more likely to be superior to the OGB graph for all types of graph-reading task types, because of its discernibilities.

\section{Perceived Usefulness}

David [25] argued that the most important determinant of technology adaptation is perceived usefulness of a technology in the Technology Acceptance Model (TAM). Perceived usefulness refers to the degree to which a person believes that using a particular system would enhance his or her job performance [25]. Perceived usefulness in graph reading context refers to the user's perception of that the graph helps them to accomplish their graph reading tasks.

Previous variations of TAM experiments have demonstrated that the user's perceived easy-of-use positively influences perceived usefulness $[25,26]$. Color is a useful addition to complicated graphs, and color often labels different graphical features so that the graph-reader can obtain relevant information quickly and easily. When the graph-reader finds the graph easier to perform various tasks, the graph is perceived more useful. Since the three lines of the OGB graph present the similar level of luminance, it is more likely to be difficult to observe one line's trend against the other two lines. This is shown as hypothesis H1.

H1: The RGB graph will be perceived more useful than the OGC graph when observing trends.

Similarly, when reading off values from a single line, the other neighboring two lines may distract the reader from concentrating the target line. Since the RGB graph shows higher contrast between a line and another, this graph is more likely to assist the user's performance of extracting values from a single line. This is shown as hypothesis $\mathrm{H} 2$.

H2: The RGB graph will be perceived more useful than the $O G C$ graph when reading a single line.

While extracting values from two lines that require the graphreader to use the two lines at the same time, distinctive colors for two lines are more likely to be helpful to compare the lines. The graph user is likely to find the high luminance contrast graph overall useful, and to believe that the color selection adds values to the graph for their easier and faster performance. Thus, this is shown as hypothesis H3.

H3: The RGB graph will be perceived more useful than the OGC graph when comparing two lines.

\section{Methodology}

The hypotheses of this study were tested through an experiment with a between-subject design. Participants were randomly assigned to two groups: RGB and OGC. The task types were designed by asking them to observe trends without extraction of values, to read a single line of Line A and Line B and two lines of the three-line graph. 


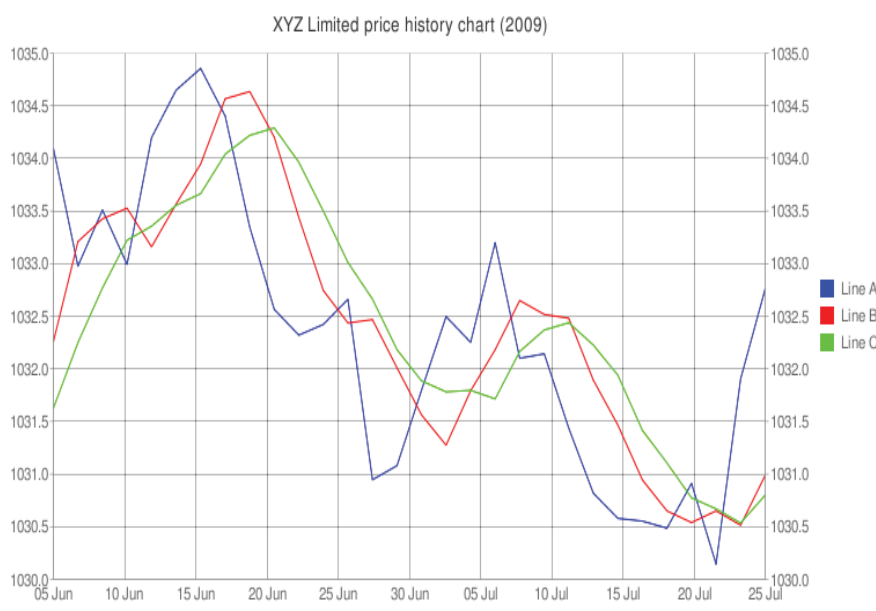

Fig. 1. A Sample Graph Presenting the RGB Combination

\section{A. Experimental Design}

The traditional RGB combination and expressive vivid-tones for a high level of luminance OGC combination were used as two groups. The three-line XYZ company's historical stock price graph was specially designed for this experiment displaying a company's historical data and the three lines were labeled with the three colors shown in Fig. 1. The size of the graph was $700 \times 450$ pixels to fit in the minimum screen resolution of $800 \times 600$ pixels. Each line of the graph was presented using $1 \mathrm{px}$ line thickness. Variations to the graph size, line thickness, and numerical scales are available to be conducted. The graph program is comparable with Internet Explorer, Mozilla Firefox, Google Chrome, Safari, and Opera web browsers to test in real life conditions. Measurements of perceived usefulness are driven from David[25]'s TAM items, as shown in Table II.

TABLE II

MEASUREMENT ITEMS

\begin{tabular}{|l|l|}
\hline H1 & $\begin{array}{l}\text { I think the color combination of the graph is useful for my tasks of } \\
\text { observing the graph trends }\end{array}$ \\
\hline H2 & $\begin{array}{l}\text { I think the color combination of the graph is useful for my tasks of } \\
\text { reading the values of a single line }\end{array}$ \\
\hline H3 & $\begin{array}{l}\text { I think the color combination of the graph is useful for my tasks of } \\
\text { figuring out the periods where Line A intersected of Line B }\end{array}$ \\
\hline
\end{tabular}

\section{B. Experimental Procedure}

A total of 41 subjects participated in the online survey from February to July 2010. The webpage of this experimental study concerning online users captured 23 female and 18 male respondents aged from 18 to 54 . The respondents generally felt confident about using computers (mean: 6.27/7) and were fairly comfortable with using graphs with numbers (mean: 5.12/7). Most of the participants (36) have no professional color training experience. Approximately one in every twelve male computer users do not see colors in the same way as the majority [18]. This survey asked the online user to identify the three line colors. This ensured all the participants' color vision was consistent and acceptable.
The each subject commenced with relatively easy graph trend assessments, reading off values, and these progressed to more advanced tasks such as calculations and interpretations from the graph. Some tasks were designed to use only a Line A approach, while other tasks were asked to use only a Line B approach. More complex tasks were also added for participants to use Line A and Line B together to compare values. The subject was asked to complete all the graph reading tasks as quickly and accurately as possible.

After completing the task session, they were asked to response to a seven-point Likert scale, ranging from 'strongly disagree (1)' to 'strongly agree (7)', about their perception of the graph user interface. Then the last part of the survey allowed the investigators to measure any individual differences that may affect the results. The participants were offered a chance to win one of ten $\$ 20$ gift vouchers as a reward.

\section{Data Analysis}

Independent T-Tests displayed in Table III and Table IV conducted on perceived-usefulness show a difference between the groups towards advanced tasks as portrayed with two lines (Line A and Line B) $(\mathrm{t}(1,39)=2.296, \mathrm{P}<.05)$. The respondents saw the RGB color combination $(\mathrm{N}=17$, mean $=$ 5.82 , s.d $=0.809$ ) as more useful than the OGC combination $(\mathrm{N}=24$, mean $=4.71$, s.d $=2.177)$. However, there are no significant differences between the groups when the subject perceived usefulness of the graphs while conducting tasks with a single line of either Line A or Line B. The level of task complexity did not affect the results in case of using a single line.

TABLE III

IndEPENDENT T-Test SumMARY FOR PERCEIVEd UseFulness

\begin{tabular}{|c|c|c|c|c|c|c|c|}
\hline & \multirow[t]{2}{*}{$\mathrm{t}$} & \multirow[t]{2}{*}{$\mathrm{df}$} & \multirow[t]{2}{*}{ Sig. } & \multirow[t]{2}{*}{$\begin{array}{l}\text { Mean } \\
\text { Diff. }\end{array}$} & \multirow[t]{2}{*}{$\begin{array}{l}\text { Std. } \\
\text { Error }\end{array}$} & \multicolumn{2}{|c|}{$\begin{array}{l}95 \% \text { Confidence } \\
\text { Interval }\end{array}$} \\
\hline & & & & & & Lower & Upper \\
\hline $\mathrm{H} 1$ & .041 & 38.99 & .968 & .025 & .600 & -1.190 & 1.239 \\
\hline $\mathrm{H} 2$ & .264 & 38.83 & .793 & .135 & .511 & -.900 & 1.169 \\
\hline H3 & 2.296 & 31.14 & .029 & 1.115 & .486 & .125 & 2.106 \\
\hline
\end{tabular}

TABLE IV

GRoup STATISTICS BETWEEN RGB AND OGC

\begin{tabular}{|c|c|c|c|c|}
\hline & & Mean & Std. Deviation & Std. Error Mean \\
\hline H1 & RGB & 4.94 & 1.600 & .388 \\
\cline { 2 - 5 } & OGC & 4.92 & 2.244 & .458 \\
\hline H2 & RGB & 5.18 & 1.402 & .340 \\
\cline { 2 - 5 } & OGC & 5.04 & 1.870 & .382 \\
\hline \multirow{2}{*}{ H3 } & RGB & 5.82 & .809 & .196 \\
\cline { 2 - 5 } & OGC & 4.71 & 2.177 & .444 \\
\hline
\end{tabular}

No significant differences were found across the two color luminance contrast conditions regarding the tasks of extracting specific values from a single line and of not extracting values but just observing big pictures of a single line trend. Means and standard deviations of perceived usefulness for each task for each group are described in Table IV. Means of perceived usefulness for observing trend tasks for the two groups are 
almost similar. There is little difference of means of perceived usefulness between the two groups for reading a single line.

Thus, there is no evidence that a high luminance contrast graph is helpful for observing and reading a single line and $\mathrm{H} 1$ and $\mathrm{H} 2$ are not supported as summarized in Table V. However, the results support a high-luminance-contrast multiple-line graph is perceived more useful than a low luminance contrast graph when extracting values from multiple lines by comparing. Hence, only $\mathrm{H} 3$ is supported as seen in Table $\mathrm{V}$.

TABLE V

Hypotheses Testing Results

\begin{tabular}{|l|c|}
\hline \multicolumn{1}{|c|}{ Hypotheses } & Results \\
\hline H1: Perceived usefulness for trends: RGB $>$ OGC & Not supported \\
\hline H2: Perceived usefulness for a single line: RGB $>$ OGC & Not supported \\
\hline H3: Perceived usefulness for two lines: RGB $>$ OGC & Supported \\
\hline
\end{tabular}

Task completion times for each task were also measured for this study. Interestingly, no significant differences were found across the color groups in terms of task completion time compared to perceived usefulness, although the respondents believe the RGB graph is useful for the two line comparing task. This explains the subject's perception and actual efficiency for their performance with the graph do not match. This will be discussed later under the future research section.

\section{DISCUSSION}

\section{A. Limitations of the Study}

This study is subject to a number of limitations. First, the color sets for graphs used red and green. Although red and green are commonly-used nominal codes in the financial charts to represent the market trends, in general, the usage of red and green is ideally not recommended for information design. McDonald [18] reported that approximately eight percent of the male population and one percent of the female population in North America and Europe is color deficient to some degree. In fact, no color deficiency was reported among the participants for this experiment, however, even if there was, the data that demonstrate kinds of color-deficiency including dichromatism, protanopia, deuteranopia, and tritanopia would have been isolated for the purpose of this study.

Second, as long as this experiment has been open to the Internet expecting that most of online users are assumed fairly competent computer users, the participants show less computer anxiety. However, during the interview process with participants who volunteered to provide feedback on the online survey, we discovered that not all participants were able to complete the survey due to the nature of the study asking number-oriented graph-reading tasks. In particular, those who have dyxlexia with numbers found the tasks challenging and simply could not continue to answer the question of reading numbers from the graph. This shows the participants may be limited to those who are familiar with using graphs.

Third, despite the moderator's role of four different task types, single-item measures were used for perceived usefulness for each task. This can increase the possibility of measurement errors. Also, ideally a larger number of participants could represent general populations.

\section{B. Future Research}

Task completion time has been a common measurement for efficiency of information systems. Most visual search studies tend to measure task completion time to assess visual attention [21]. Although the graph reading context differs from visual search studies, a salient feature has become an important role of holding the user's attention for designed tasks with the information design. If the user finds the target item faster, it is intrinsically assumed in the searching-item context, the target item grabs the user's attention more.

Nominal codes for graphs often require some degree of holding the graph-reader's attention to match the graphical cues with variables quickly and easily. The graph-reader may find alerting color contrast for graph labels useful for their tasks but the results of this study demonstrates there is no actual difference of task completion time regarding color contrast. Then perceived usefulness should be understood in the context of not only objective efficiency but also the graphuser's subjective affective states while using the graph.

In general, high contrast graphs are believed functional but other sets of color combination such as low contrast of vividtone luminance and medium level of luminance like magenta, pink, and sky blue. In fact, these vivid-tone labels are widely substituted for a traditional high contrast RGB set as nominal codes for graphs in real life as hue contrast combination. Other factors of visual aesthetics, likeability, and overall satisfaction should be explored with the relationship of visual attention and perceived usefulness towards graphs.

Furthermore, as discussed in the previous section, the space of color nominal codes for line graph is limited. The principle of color perception may be applied in a different way to bar graphs and pie charts that display comparatively larger portions for color codes than line graphs. This research can be further expanded using varied types of graphs and different types of graph-reading tasks.

The major issues of color selection for information displays are clarity and comprehensibility. How easy to distinguish a color cue from its background and how obvious a color code is relative to its neighbors depend on the nature of graphs. The surrounding designs of sizes, the interval of grid systems, and layouts may affect the graph-reader's perception of color labels. Information designers can manipulate each visual design factors and they are possibly able to explore the most efficient and satisfactory graphs.

Lastly, the number of multiple lines should be examined for further studies. Color graph studies have been rare and moreover multiple color line graphs studies have been seldom seen so far. Considering the vital role of color nominal codes for multiple line graphs and a massive usage of line graphs in everyday life, the variations of the number of multiple color lines are worth examining as laboratory experiments or with actual online computer users. 


\section{CONCLUSION}

This study on multiple color combinations explores the user's perceived levels of usefulness of the graph depending on task types. This study contributes in three areas. First, the comparison of color combinations, as an aspect of graph comprehension, is demonstrated. Second, this study examines objective usability of task completion time as well as the user's subjective perceived usefulness. Third, this research investigates the moderating effects of task types with the color information presentation. This aspect has rarely received attention by researchers, particularly the connection between perceived usefulness and color luminance contrast and the complexity of task types.

\section{ACKNOWLEDGMENT}

This research is sponsored by the Sejong Foundation of Korea and the Faculty of Law, Business, and the Creative Arts of James Cook University through Higher Degree Research Grant (2009).

\section{REFERENCES}

[1] P. Mather, A. Ramsay, and A. Serry, "The Use and Representational Faithfulness of Graphs in Annual Reports: Australian Evidences," Australian Accounting Review, vol. 6, pp. 56-63, 1996.

[2] J.M. Penrose, "Annual Report Graphic Use: A Review of the Literature," Journal of Business Communication, vol. 45, pp. 158-180, 2008.

[3] S.B. Trickett and J.G. Trafton, "Toward a Comprehensive Model of Graph Comprehension: Making the Case for Spatial Cognition," in Diagrams, D. Barker-Plummer, Ed. Berlin Heidelberg: SpringerVerlag, 2006, pp. 286-300.

[4] M. Stone, A Field Guide to Digital Color. Natick: A K Peters, Ltd., 2008.

[5] C. Ware, Information Visualization: Perception for Design, 2nd ed. San Francisco: Morgan Kaufmann, 2004.

[6] M. Stone, "Color in Information Display," in Vis06, 2006.

[7] J.R. Stroop, "Studies of Interference in Serial Verbal Reactions," Journal of Experimental Psychology, vol. 18, pp. 643-662, 1935.

[8] Australia Stock Exchange, http://www.asx.com.au/asx/research/companyInfo.do?by=asxCode\&asx Code $=$ ASX

[9] Google Finance, http://www.google.com/finance?q=INDEXDJX:.DJI,INDEXSP:.INX,I NDEXNASDAQ:.IXIC

[10] Bloomberg, http://www.bloomberg.com/index eu.html

[11] L.C. Ou and M.R. Luo, "A Color Harmony Model for Two-Color Combinations," Color Research and Application, vol. 31, pp. 191-204, 2006.

[12] I. Benbasat and A.S. Dexter, "An Investigation of the Effectiveness of Color and Graphical Information Presentation under Varying Time Constraints," MIS Quarterly, vol. 10, pp. 59-83, March 1986.

[13] N. Kumar and I. Benbasat, "The Effect of Relationship Encoding, Task Type, and Complexity on Information Representation: An Empirical Evaluation of 2D and 3D Line Graphs," MIS Quarterly, vol. 28, pp. 255-281, June 2004

[14] D. Peebles and P.C.H. Cheng, "Modeling the Effect of Task and Graphical Representation on Response Latency in a Graph Reading Task," Human Factors, vol. 45, pp. 28-46, 2003.

[15] E.R. Tufte, "Envisioning Information," Graphics Press, Cheshire, Cnnecticut, 1998.

[16] L. Arend, "Legibility," in Using Color in Information Display Graphics: Color Usage Research Lab, NASA Ames Research Center, 2010.
[17] M.D. Fairchild, Color Appearance Models, 2nd ed. Hoboken: John Wiley \& Sons, Ltd, 2005.

[18] L.W. MacDonald, "Using Color Effectively in Computer Graphics," IEEE Computer Graphics and Applications, pp. 20-35, July/August 1999.

[19] E. Hering, "Outlines of a Theory of Light Sense," Springer-Verlag, 1920 .

[20] I.E. Gordon, Theories of Visual Perception, 3rd ed. New York: Psychology Press, 2004.

[21] J.H.R. Maunsell and S. Treue, "Feature-Based Attention in Visual Cortex," Trends in Neurosciences, vol. 29, pp. 317-322, 2006.

[22] W. Hong, J.Y.L. Thong, and K.Y. Tam, "Does Animation Attract Online Users' Attention?: The Effects of Flash on Information Search Performance and Perceptions," Information Systems Research, vol. 15, pp. 60-86, March 2004.

[23] T. Sutton and B.M. Whelan, The Complete Color Harmony: Leads Press, 2008

[24] C. Speier and M.G. Morris, "The Influence of Query Interface Design on Decision-Making Performance," MIS Quarterly, vol. 27, pp. 397423, September 2003.

[25] F.D. Davis, "Perceived Usefulness, Perceived Ease of Use, and User Acceptance of Information Technology," MIS Quarterly, vol. 13, pp. 319-339, 1989.

[26] H.v.d. Heijden, "User Acceptance of Hedonic Information Systems," MIS Quarterly, vol. 28, pp. 695-704, 2004. 